Abstract

\title{
Complexity as Cardiorespiratory Coupling Measure in Neonates with Different Gestational Ages ${ }^{\dagger}$
}

\author{
Maria Ribeiro 1,2, Luísa Castro 1,3, Luís Antunes 1,2, Cristina Costa-Santos ${ }^{3,4}$ and Teresa Henriques ${ }^{3,4}$ \\ 1 Center for Research in Advanced Computing Systems, Institute for Systems and Computer Engineering, \\ Technology and Science (CRACS/INESC-TEC), Porto, Portugal \\ 2 Computer Science Department, Faculty of Sciences, University of Porto, Porto, Portugal \\ 3 Centre for Health Technology and Services Research (CINTESIS), Faculty of Medicine University of Porto, \\ Porto, Portugal \\ 4 Health Information and Decision Sciences Department-MEDCIDS, Faculty of Medicine, University of Porto, \\ Porto, Portugal \\ + Presented at the Entropy 2021: The Scientific Tool of the 21st Century, 5-7 May 2021; Available online: \\ https://sciforum.net/conference/Entropy2021/.
}

Published: 5 May 2021

After the transition from fetal to neonatal life, the cardio-respiratory system needs to adapt to the extrauterine condition. Both the cardiac and respiratory systems display complex dynamics. This study aimed to investigate the relationship between cardiorespiratory coupling, heart rate variability (HRV), and respiration of neonatal with gestational age (GA). Several complexity measures have been developed to quantify the complexity of physiological signals. In this study, we applied sample entropy (SampEn) and the bzip2 compressor to the time series. The mutual information (MI) and the normalized compression distance (NCD) were used to quantify the complexity of the cardiorespiratory coupling.

We analyzed a dataset composed of 30-minutes traces of RR intervals and respiration signals, acquired in the first two days of life, for 33 neonates with GA between 27 and 41 weeks. Of these 33 neonates, 22 babies were premature ( $<37$ weeks), and 4 babies were considered extremely premature ( $<28$ weeks). The Pearson correlation was computed to assess the association between complexity measures and GA.

Results obtained show that for the respiratory signals, SampEn increases as GA increases ( $r=0.46$, $\mathrm{p}=0.008$ ). However, the SampEn for RR intervals and MI gave non-significant correlations. When we applied the bzip2 compressor to the RR signals, we obtained a positive correlation with GA ( $r=0.69$, $\mathrm{p}<0.001$ ), but there is no significant correlation between bzip2 of respiratory signals and GA. For the complexity of cardiorespiratory coupling with $\mathrm{NCD}$, we obtained a negative correlation with GA $(\mathrm{r}=-0.74, \mathrm{p}<0.001)$.

We infer that SampEn presents better results for respiratory signals. However, bzip2 is better when using RR signals. While the complexity of the time series increases with GA, the complexity of the coupling decreases. This finding might emerge from the fact that the heart rate is highly modulated by respiration in premature babies. Future studies should investigate the complementary of these complexity measures.

(C) 2021 by the authors. Licensee MDPI, Basel, Switzerland. This article is an open access article distributed under the terms and conditions of the Creative Commons Attribution (CC BY) license (http://creativecommons.org/licenses/by/4.0/). 